\title{
On a general degenerate/singular parabolic equation with a nonlocal space term
}

\author{
Brahim Allal ${ }^{1}$, Genni Fragnelli ${ }^{2}$, and Jawad Salhi ${ }^{3}$ \\ ${ }^{1}$ Universite Hassan 1er de Settat \\ ${ }^{2}$ Università degli Studi di Bari \\ ${ }^{3}$ Moulay Ismail University of Meknes
}

December 11, 2021

\begin{abstract}
In this paper we study the null controllability for the problems associated to the operators $\mathrm{y} \_t_{-} \mathrm{Ay}-\backslash \mathrm{lambda} / \mathrm{b}(\mathrm{x}) \mathrm{y}+\backslash \mathrm{int} \_0 \wedge 1$ $\mathrm{K}(\mathrm{t}, \mathrm{x}, \backslash$ tau $) \mathrm{y}(\mathrm{t}, \backslash \mathrm{tau}) \mathrm{d} \backslash \mathrm{tau},(\mathrm{t}, \mathrm{x}) \backslash$ in $(0, \mathrm{~T}) \backslash$ times $(0,1)$ where Ay $:=\mathrm{ay}_{-}\{\mathrm{xx}\}$ or Ay :=(ay_x $)_{-} \mathrm{x}$ and the functions a and $\mathrm{b}$ degenerate at an interior point $\mathrm{x} 0$ Ë .0; 1 . To this aim, as a first step we study the well posedness, the Carleman estimates and the null controllability for the associated nonhomogeneous degenerate and singular heat equations. Then,using the Kakutani's fixed point Theorem, we deduce the null controllability property for the initial nonlocal problems.
\end{abstract}

\section{Hosted file}

AFS2021.pdf available at https://authorea.com/users/450696/articles/548995-on-a-generaldegenerate-singular-parabolic-equation-with-a-nonlocal-space-term 